\title{
Thermal and mechanical properties of the near-surface layers of comet 67P/Churyumov-Gerasimenko
}

\section{T. Spohn, ${ }^{1 *}$ J. Knollenberg, ${ }^{1}$ A. J. Ball, ${ }^{2}$ M. Banaszkiewicz, ${ }^{3}$ J. Benkhoff, ${ }^{2}$ M. Grott, ${ }^{1}$ J. Grygorczuk, ${ }^{3}$ C. Hüttig, ${ }^{1}$ A. Hagermann, ${ }^{4}$ G. Kargl, ${ }^{5}$ E. Kaufmann, ${ }^{4}$ N. Kömle, ${ }^{5}$ E. Kührt, ${ }^{1}$ K. J. Kossacki, ${ }^{6}$ W. Marczewski, ${ }^{3}$ I. Pelivan, ${ }^{1}$ R. Schrödter, ${ }^{1}$ K. Seiferlin ${ }^{7}$}

Thermal and mechanical material properties determine comet evolution and even solar system formation because comets are considered remnant volatile-rich planetesimals. Using data from the Multipurpose Sensors for Surface and Sub-Surface Science (MUPUS) instrument package gathered at the Philae landing site Abydos on comet 67P/Churyumov-Gerasimenko, we found the diurnal temperature to vary between 90 and $130 \mathrm{~K}$. The surface emissivity was 0.97 , and the local thermal inertia was $85 \pm 35 \mathrm{~J} \mathrm{~m}^{-2} \mathrm{~K}^{-1} \mathrm{~s}^{-1 / 2}$. The MUPUS thermal probe did not fully penetrate the near-surface layers, suggesting a local resistance of the ground to penetration of $>4$ megapascals, equivalent to $>2$ megapascal uniaxial compressive strength. A sintered near-surface microporous dust-ice layer with a porosity of 30 to $65 \%$ is consistent with the data.

I he Multipurpose Sensors for Surface and Sub-Surface Science (MUPUS) package $(1,2)$ operated on the approach to and on the surface of 67P/Churyumov-Gerasimenko (67P) between 12 and 14 November 2014. MUPUS (Fig. 1) is composed of three separate instruments: (i) a thermal probe, MUPUS-PEN, which is equipped with 16 resistance temperature detector-type titanium temperature sensors to be inserted into the cometary surface by a hammer mechanism; (ii) an infrared radiometer, MUPUS-TM; and (iii) a thermal sensor and an accelerometer in each of the two harpoon anchors of the lander. MUPUS-PEN was stowed on Philae during flight, and landing and was nominally deployed. MUPUS-TM is mounted to the lander in a fixed position, with the line of sight having an angle of $45^{\circ}$ relative to the lander vertical axis and an opening angle of $\pm 30^{\circ}$ around the line of sight. The total field of view (FOV) of $\sim 1 \mathrm{~m}^{2}$ and the full-width-half-maximum FOV of $0.3 \mathrm{~m}^{2}$ include the MUPUS-PEN deployment location.

MUPUS was designed to measure thermal and mechanical properties of the surface and the nearsurface layers and to monitor the subsurface temperature. Because the harpoon anchors failed to fire (for reasons still being investigated by the lander engineers) and because MUPUS-PEN could not be fully inserted, subsurface thermal and mechanical properties could not be measured, unfortunately. Instead, a lower bound on the

${ }^{1}$ Institute of Planetary Research, Deutsches Zentrum für Luft- und Raumfahrt (DLR), Berlin, Germany. ${ }^{2}$ European Space Research and Technology Centre (ESTEC), European Space Agency (ESA), Noordwijk, Netherlands. ${ }^{3}$ Space Research Center, Warsaw, Poland. ${ }^{4}$ Department of Physical Sciences, The Open University, Milton Keynes, UK. ${ }^{5}$ Space Research Institute, Austrian Academy of Sciences Graz, Austria. ${ }^{6}$ Faculty of Physics, University of Warsaw, Warsaw, Poland. ${ }^{7}$ Physics Institute, University of Berne, Berne, Switzerland

*Corresponding author. E-mail: tilman.spohn@dlr.de strength of the subsurface material was derived from the observed failure to penetrate of MUPUSPEN. MUPUS-TM data were inverted in order to calculate the local daily temperature variation, the thermal emissivity, and the thermal inertia.

MUPUS-TM was switched on 1 hour before the release of Philae from Rosetta and recorded during the descent of the lander and its involuntary flight across the surface until final touchdown. These data include coverage of the surface along the flight path, which need to be evaluated separately. The MUPUS-TM data have been used to help reconstruct the flight path of the lander (3). After the lander had settled at its final landing site, Abydos, MUPUS-TM recorded for another 41 hours.

The diurnal temperature variation (Fig. 2) was synthesized from 3 days of radiometer data and from MUPUS-PEN temperature recordings (figs. S2 to $\mathrm{S} 4$ ). The emissivity $\varepsilon$ was determined by means of least-square analysis of the TM data and was found to be $\sim 0.97$. The temperature varies between 90 and $130 \mathrm{~K}$. This is an equivalent graybody temperature. It should be representative of the average temperature in the FOV of MUPUSTM. Also shown is our estimate of the $2 \sigma$ uncertainty range. A characteristic peak in temperature of $\sim 36$ min duration is shown in Fig. 2. The complete temperature history recordings at Abydos (figs. S2 and S4), derived from the flux measured with MUPUS-TM and from MUPUS-PEN temperature recordings, show three peaks separated by 12.4 hours, which is the rotation period of the nucleus (4). We interpret these peaks as infrared radiation from the directly insolated surface, whereas the more gentle variation of the temperature outside a peak is interpreted as being due to indirect lighting.

Thermal models have been calculated to fit the data (Fig. 2). The calculation is described in more detail in the supplementary materials. We show three curves for thermal inertia $I=\sqrt{k \rho c}$, where $k$ is the thermal conductivity, $\rho$ is the density, and $c$ is the specific heat, varying between 50 and $120 \mathrm{~J} \mathrm{~m}^{-2} \mathrm{~K}^{-1} \mathrm{~s}^{-1 / 2}$, with $85 \mathrm{~J} \mathrm{~m}^{-2} \mathrm{~K}^{-1} \mathrm{~s}^{-1 / 2}$ providing the best fit. A thermal inertia of 10 to

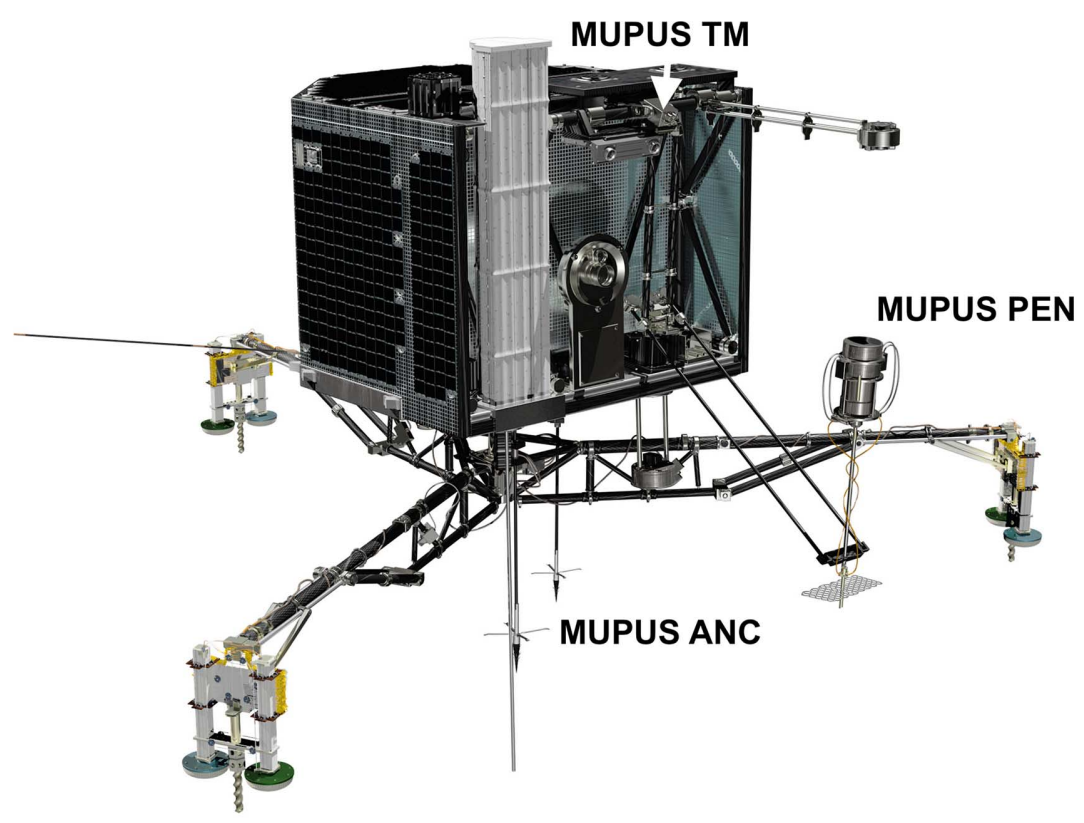

Fig. 1. Elements of the MUPUS package on the Rosetta lander Philae. Shown is Philae with the instrument bench (also termed the balcony). The thermal mapper MUPUS-TM is shown along with the deployed thermal probe MUPUS-PEN and the two anchors, each of which houses an accelerometer, MUPUS ANC-M, and a temperature sensor, ANC-T. MUPUS-PEN is shown connected to its deployment device extending from the balcony. This double strut device was retracted 300 min after MUPUS switched on so as to allow a later rotation of the lander body. [Image courtesy ESA/ATG media lab] 
$50 \mathrm{~J} \mathrm{~m}^{-2} \mathrm{~K}^{-1} \mathrm{~s}^{-1 / 2}$ has been inferred from the measurements reported by the Microwave Instrument for the Rosetta Orbiter (MIRO) as a representative value of the overall surface of 67P (5). Our value is clearly larger, although considering the variability of the parameters determining the thermal inertia, not dramatically so. Abydos may simply have a thinner spread of low-conductivity and -density dust than on average, underlain by a sintered but still porous dust-ice layer. Other differences may be in the dust-to-ice ratio, dust composition, and local versus global average temperature and subsurface temperature gradients. MIRO as a millimeter and submillimeter instrument samples a thicker layer than does MUPUS-TM. From unresolved Spitzer observations of 67P, an upper limit of $15 \mathrm{~J} \mathrm{~m}^{-2} \mathrm{~K}^{-1} \mathrm{~s}^{-1 / 2}$ was proposed (6). Other estimates of the thermal inertia of cometary nuclei are $<45$ and $200 \mathrm{~J} \mathrm{~m}^{-2} \mathrm{~K}^{-1} \mathrm{~s}^{-1 / 2}(7,8)$ for comet 9P/Tempel 1-both studies using the same Deep Impact data-and $<250 \mathrm{~J} \mathrm{~m}^{-2} \mathrm{~K}^{-1} \mathrm{~S}^{-1 / 2}(7)$ for comet Hartley 2. Our value is smaller than that of kilometer-sized near-Earth asteroids (9) and suggests a near-surface porous layer. Differences in thermal inertia may further indicate differences in degrees of compaction and sintering and concentrations of organics in near-surface layers (10).

Unfortunately, the local values of $k, \rho$, and $c$ have not been individually measured (11). Using the overall density of the comet of $470 \pm 45 \mathrm{~kg} \mathrm{~m}^{-3}$ (4) and a specific heat of 300 to $600 \mathrm{~J} \mathrm{~kg}^{-1} \mathrm{~K}^{-1}$ as appropriate for a mixture of silicate dust (12) and water ice (13), with a dust-to-ice ratio of 2 to 6 (14) at temperatures of 90 to $130 \mathrm{~K}$, a thermal conductivity of 0.02 to $0.06 \mathrm{~W} \mathrm{~m}^{-1} \mathrm{~K}^{-1}$ is implied for $I=85 \mathrm{~J} \mathrm{~m}^{-2} \mathrm{~K}^{-1} \mathrm{~s}^{-1 / 2}\left(8 \times 10^{-3}\right.$ to $0.11 \mathrm{~W} \mathrm{~m}^{-1} \mathrm{~K}^{-1}$ if the full uncertainty of the thermal inertia is considered). These values compare with values of $2 \times 10^{-3}$ to $0.02 \mathrm{~W} \mathrm{~m}^{-1} \mathrm{~K}^{-1}$ reported for silicate dust in vacuum (15), albeit at ambient temperatures, and with values of 0.02 to $0.03 \mathrm{~W} \mathrm{~m}^{-1} \mathrm{~K}^{-1}$ for nonsintered porous ice at $100 \mathrm{~K}(16)$. Our values are consistent with porosities between 40 and $55 \%$ (30 to $65 \%$ for the full range of uncertainty), using the data from (15). The thermal conductivity of highly porous media depends more on the porosity and on the contact area between the grains than on the bulk thermal conductivity of the matrix material $(15,16)$.

The MUPUS-PEN probe was nominally commanded to start the hammering sequence $40 \mathrm{~min}$ after it had been deployed. The depth-of-penetration sensor, DS, measured the progress of the hammering. The hammering was also recorded by the three accelerometers of the Surface Electrical, Seismic and Acoustic Monitoring Experiment (SESAME) installed in the feet of the lander (17). The DS recorded an initial progress of $\sim 27 \mathrm{~mm}$, and then its readings oscillated for 3.5 hours by a total of 10 to $15 \mathrm{~mm}$, indicating no net progress on penetration (Fig. 3). The rapid initial penetration could have been through a thin layer of dust, but this is speculative in the absence of precise knowledge of the initial height of the PEN tip above the ground and other independent evidence for a dust layer at Abydos. Hammering started with the lowest energy setting (Table 1 and supplementary materials). Because progress measured by the DS was smaller than a threshold value of $1.7 \mathrm{~mm}$, the energy level was increased by one level after the other and had been increased after a total of 56 strokes to its maximum, where it remained for 3.1 hours. The evidence for failure to penetrate comes foremost from the recording of the DS but is supported by the temperatures measured along the PEN (fig. S4). These temperatures follow the temperature changes recorded by the thermal mapper, in particular, when the environment is warmed by indirect light.

The lack of progress into the subsurface can be interpreted as being caused by a near-surface layer of a strength the PEN was not capable of penetrating. Alternative interpretations invoke malfunctioning of MUPUS-PEN but are not independently confirmed. These are discussed in the supplementary materials. It is very probable that the mechanism worked nominally at least for three of the four levels. For level 4, housekeep-
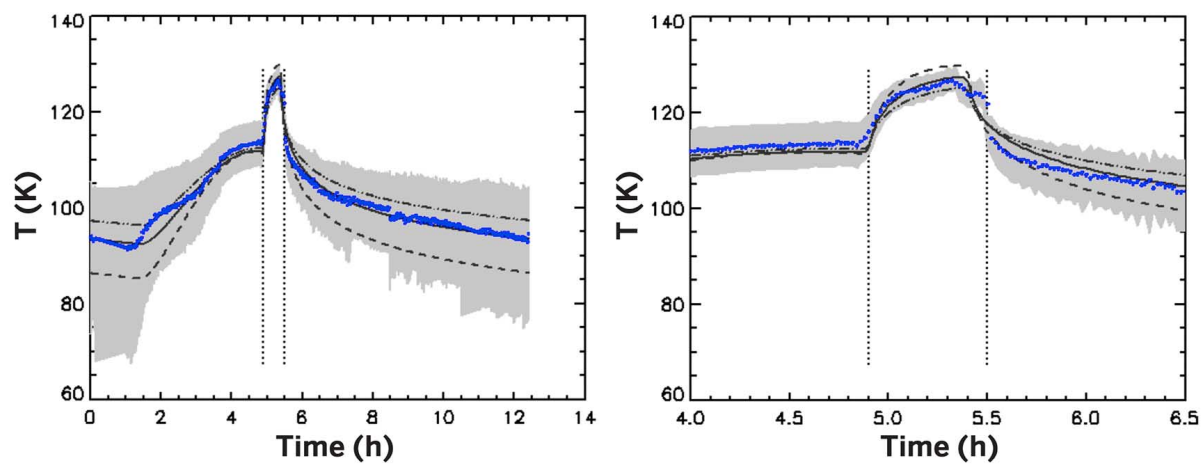

Fig. 2. Variation of temperature during a comet day at Abydos. The temperature record (blue) was synthesized from 3 days of radiometer data. (Left) The diurnal variation. (Right) The temperature during a 40-min direct illumination of part of the FOV of the thermal mapper. The gray area indicates the $2 \sigma$ uncertainty estimate. The model calculations are for a thermal inertia of $85 \mathrm{~J} \mathrm{~m}^{-2} \mathrm{~K}^{-1} \mathrm{~s}^{-1 / 2}$ (solid line), a ratio between stray light and direct illumination of $\beta_{0}=0.32$, and $69 \%$ of the FOV illuminated. The dashed line is for $50 \mathrm{~J} \mathrm{~m}^{-2} \mathrm{~K}^{-1} \mathrm{~s}^{-1 / 2}, \beta_{0}=0.27$, and $46 \%$ of the FOV illuminated, and the dash-dotted line is for $120 \mathrm{~J} \mathrm{~m}^{-2} \mathrm{~K}^{-1} \mathrm{~s}^{-1 / 2}, \beta_{\mathrm{O}}=0.35$, and $80 \%$ of the FOV illuminated. Details of the calculation are provided in the supplementary materials.

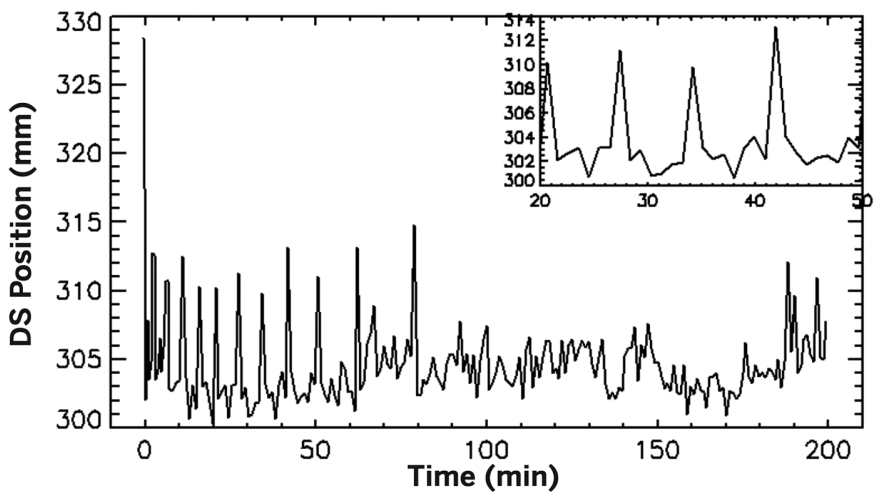

Fig. 3. Displacement of the MUPUS-PEN depth sensor (DS). The sensor records the progress of penetration with MUPUS-PEN. There is a clear downward motion of $27 \mathrm{~mm}$ at the beginning, followed by oscillatory displacements by 10 to $15 \mathrm{~mm}$, followed by smaller displacements. The reason for the reduction in amplitude after time $(t)=80 \mathrm{~min}$ is not understood. One possibility is that the barbs at the tip of the PEN locked to the ground. Overall, it appears as though the PEN was hammering more or less on the spot (but not necessarily at exactly the same spot), with indentations of a few millimeters and recoils of up to $10 \mathrm{~mm}$. 
Table 1. PEN hammer calibration data. The table collects PEN hammer calibration results in three foam glasses of differing strength. Foam glasses T4 and $\mathrm{F}$ are commercially available. Foam glass SRC was produced at Space Research Centre (SRC) Warsaw for the prelaunch calibration measurements. The samples were kept for reference at SRC Warsaw. Listed are the uniaxial compressive strengths as given by the manufacturers for foam glasses $\mathrm{T} 4$ and $\mathrm{F}$, and the results of control measurements done at Technische Universität (TU) Graz in 2015 for foam glasses F and SRC along with a value calculated for T4 (22) using data of a shear deformation measurement. Also given is the static penetration resistance measured at SRC Warsaw (supplementary materials).
The static penetration resistance per unit area is the strength of a material into which a pile is driven. The compressive strength is measured by placing a sample cylinder between two plates of the same radius. For a homogenous, isotropic medium and a thin pile, the static penetration resistance per unit area should be twice the uniaxial compressive strength for geometrical reasons of stress propagation. The table further gives the rate of penetration progress in millimeters per four hammer strokes at the four energy levels of the hammer mechanism. The energy stored in the capacitor of the mechanism is listed per level. The efficiency of the PEN mechanism, defined as the ratio of deformational energy to the energy stored, was found to be $\sim 10 \%$.

\begin{tabular}{|c|c|c|c|}
\hline & Foam glass T4 & Foam glass $\mathbf{F}$ & Foam glass SRC \\
\hline Compressive strength (MPa) (nominal by manufacturer) & 0.85 & 1.70 & - \\
\hline Uniaxial compressive strength (MPa) & $0.23^{*}$ & $0.52^{\dagger}$ & $2.11^{+}$ \\
\hline Static penetration resistance per unit area of pile (MPa) (SRC, 2015) & $0.52 \pm 0.09$ & $1.24 \pm 0.32$ & $4.19 \pm 1.05$ \\
\hline \multicolumn{4}{|l|}{ PEN hammer calibration results } \\
\hline \multicolumn{4}{|l|}{ Progress per four hammer strokes (mm) } \\
\hline Level $1,0.49 \mathrm{~J}$ & 2.1 & & \\
\hline Level 2, $1.59 \mathrm{~J}$ & 4.8 & 2.6 & \\
\hline Level 3, $2.17 \mathrm{~J}$ & 8.3 & 4.4 & 0.3 \\
\hline Level 4, $4.23 \mathrm{~J}$ & & & 2.0 \\
\hline
\end{tabular}

*From Kömle et al. (22). †TU Graz, 2015. same sample; error estimates are $2 \sigma$. $\ddagger$ Average values from four measurements are described in more detail in the supplementary materials, each with the
(Table 1) and an opening angle of the PEN tip o of $28.5^{\circ}$, the force per unit area of tip surface to overcome the strength is $\sigma=\chi E \cos ^{2} \alpha / \delta^{2} \sin \alpha$. Considering only energy level 3 , we find a value of $7 \mathrm{MPa}$. Given the uncertainty of the exercisein particular, in the deformation $\delta$-we take this value as a confirmation of the above lower bound of the resistance to penetration of $4 \mathrm{MPa}$.

Deep Impact crater observation for comet Tempel-1 resulted in a tensile strength estimate of $<12 \mathrm{kPa}$ (18). Observation of cometary meteoroids suggest tensile strengths of $\sim 10 \mathrm{kPa}$ for particles that originated from pristine comets and up to $80 \mathrm{kPa}$ for those from evolved comets (such as taurids from $2 \mathrm{P} /$ Encke) (19). Tensile strengths are typically at least one order of magnitude smaller than compressive strengths (20).

Laboratory data on the strength of ice and ice dust mixtures are rare at relevant temperatures as measured at Abydos. Granular ice of $1 \mathrm{~mm}$ grain size has a compressive strength of 60 to $70 \mathrm{MPa}$ at 100 to $150 \mathrm{~K}(21)$, which is an order of magnitude larger than our lower bound. The static penetration resistance of sintered porous ice with an initial porosity of $73 \%$ is $10 \mathrm{MPa}$ at $220 \mathrm{~K}$, whereas the static penetration resistance of sintered $\mathrm{CO}_{2}$ ice with an initial porosity of $48 \%$ is $6.5 \mathrm{MPa}$ at $193 \mathrm{~K}(22)$. Moreover, sintering of highly porous ice during the Comet Simulation (KOSI) experiments (23-25) resulted in a penetration resistance of $5 \mathrm{MPa}(24)$. These values compare quite well with our estimates and with our independent estimate of the porosity from the MUPUS-TM data. According to a comet thermal evolution model, sintering can result in a strong near-surface layer of a comet nucleus (26) but requires the grains to be tens of micrometers in radius, or smaller. Thus, high strength suggests that the material is at least locally fine-grained.
Both the thermal inertia and the strength on the surface of $67 \mathrm{P}$ are larger than commonly thought. In particular, our lower bound on the strength of the subsurface material is larger than previous estimates from the stability of largescale features both on $67 \mathrm{P}(27)$ and on other comets. The reason may lie with layering or with largescale cracks in the nucleus that may render parts of it unstable. From our data, we envisage the nucleus as a low-thermal conductivity, highly porous body with a near-surface layer consisting of sintered ice-dust with substantial local strength. The layer could be covered by a thin layer of dust of low strength. The estimated porosity is consistent with earlier models of comet nucleus formation $(28,29)$.

\section{REFERENCES AND NOTES}

1. T. Spohn et al., MUPUS-A thermal and mechanical properties probe for the Rosetta lander Philae. Space Sci. Rev. 128 339-362 (2007). doi: 10.1007/s11214-006-9081-2

2. MUPUS was originally developed at the Institute of Planetology of the University of Münster together with the Space Research Center in Warsaw, the DLR Institute of Planetary Research, the Space Research Institute Graz, the University of Kent, and the Open University, and other international partners. It is now maintained and operated by an international team led by the DLR Institute of Planetary Research in Berlin.

3. J. Biele et al., The landing(s) of Philae and inferences about comet surface mechanical properties. Science $\mathbf{3 4 9}$, aaa9816 (2015)

4. H. Sierks et al., On the nucleus structure and activity of comet 67P/Churyumov-Gerasimenko. Science 347, aaa1044 (2015). doi: 10.1126/science.aaa1044; pmid: 25613897

5. S. Gulkis et al., Subsurface properties and early activity of comet 67P/Churyumov-Gerasimenko. Science 347, aaa0709 (2015). doi: 10.1126/science.aaa0709; pmid: 25613896

6. S. Lowry et al., The nucleus of Comet 67P/ChuryumovGerasimenko - A new shape model and thermophysical analysis. Astron. Astrophys. 548, A12 (2012)

7. 0 . Groussin et al.. The temperature, thermal inertia, roughness and color of the nuclei of Comets 103P/Hartley 2 and 9P/Tempel 1. Icarus 222, 580-594 (2013). doi: 10.1016/ j.icarus.2012.10.003
8. B. J. R. Davidsson, P. J. Gutiérrez, H. Rickmann, Physical properties of morphological units on comet 9P/Tempel 1 derived from near-IR Deep Impact spectra. Icarus 201, 335-357 (2009). doi: 10.1016/j.icarus.2008.12.039

9. M. Delbo, A. dell'Oro, A. W. Harris, S. Mottola, M. Mueller, Thermal inertia of near-Earth asteroids and implications for the magnitude of the Yarkovsky effect. Icarus 190, 236-249 (2007). doi: 10.1016/j.icarus.2007.03.007

10. B. J. R. Davidsson et al., Thermal inertia and surface roughness of Comet 9P/Tempel 1. Icarus 224, 154-171 (2013). doi: 10.1016/j.icarus.2013.02.008

11. A densitometer originally planned to be included with MUPUS had to be descoped for lack of funding. The direct measurement of the thermal conductivity with the PEN was not performed because the PEN had not been inserted and time was lacking.

12. D. F. Winter, J. M. Saari, A particulate thermophysical model of the lunar soil. Astrophys. J. 156, 1135-1151 (1969). doi: 10.1086/150041

13. G. Herman, P. R. Weissman, Numerical simulation of cometary nuclei: III. Internal temperatures of cometary nuclei. Icarus 69 314-328 (1987). doi: 10.1016/0019-1035(87)90108-4

14. A. Rotundi et al., Dust measurements in the coma of comet 67P/Churyumov-Gerasimenko inbound to the Sun. Science 347, aaa3905 (2015). doi: 10.1126/science.aaa3905 pmid: 25613898

15. M. Krause, J. Blum, Yu. V. Skorov, M. Trieloff, Thermal conductivity measurements of porous dust aggregates: I. Technique, model and first results. Icarus 214, 286-296 (2011). doi: 10.1016/j.icarus.2011.04.024

16. K. Seiferlin, N. Kömle, G. Kargl, T. Spohn, Line heat-source measurements of the thermal conductivity of porous $\mathrm{H}_{2} \mathrm{O}$ ice, $\mathrm{CO}_{2}$ ice and mineral powders under space conditions. Planet. Space Sci. 44, 691-704 (1996). doi: 10.1016/ 0032-0633(96)00068-2

17. K. J. Seidensticker et al., SESAME-An experiment of the Rosetta lander Philae: Objectives and general design Space Sci. Rev. 128, 301-337 (2007).

18. K. A. Holsapple, K. R. Housen, A crater and its ejecta: An interpretation of Deep Impact. Icarus 187, 345-356 (2007). doi: 10.1016/j.icarus.2006.08.029

19. J. M. Trigo-Rodriguez, J. Blum, Tensile strength as an indicator of the degree of primitiveness of undifferentiated bodies. Planet. Space Sci. 57, 243-249 (2009). doi: 10.1016/j.pss.2008.02.011

20. J. Biele et al., The putative mechanical strength of comet surface material applied to landing on a comet. Acta Astronaut. 65, 1168-1178 (2009). doi: 10.1016/j.actaastro.2009.03.041

21. R. M. Schulson, P. Duval, Creep and Fracture of Ice (Cambridge Univ. Press, Cambridge, 2009). 
22. N. Kömle et al., Impact penetrometry on a comet nucleus-Interpretation of laboratory data using penetration models. Planet. Space Sci. 49, 575-598 (2001). doi: 10.1016/S0032-0633(00)00169-0

23. KOSI experiments were performed between 1987 and 1993 in the space simulator at DLR in Cologne/Germany and are described in $(24,25)$. The experiments placed a sample of granular ice at liquid nitrogen temperatures in a vacuum chamber. The samples were insolated mostly with one solar constant, but the insolation was varied between experiments. The thermal histories and the modifications of the ice-in particular, sublimation and sintering-were observed and documented.

24. E. Grün, H. Kochan, K. J. Seidensticker, Laboratory simulation, a tool for comet research. Geophys. Res. Lett. 18, 245-248 (1991). doi: 10.1029/90GL02522

25. H. W. Kochan, W. F. Huebner, D. W. G. Sears, Simulation experiments with cometary analogous material. Lab.
Astrophys. Space Res. 236, 623-665 (1999). doi: 10.1007/ 978-94-011-4728-6_25

26. K. J. Kossacki, Comet 9P/Tempel 1: Evolution of the surface. Icarus 245, 348-354 (2015). doi: 10.1016/ j.icarus.2014.09.044

27. N. Thomas et al., The morphological diversity of comet 67P/Churyumov-Gerasimenko. Science 347, aaa0440 (2015). doi: 10.1126/science.aaa0440; pmid: 25613893

28. J. Blum, R. Schräpler, B. J. R. Davidsson, J. M. Trigo-Rodriguez, The physics of protoplanetesimal dust agglomerates.

I. Mechanical properties and relations to primitive bodies in the solar system. Astrophys. J. 652, 1768-1781 (2006). doi: 10.1086/508017

29. J. Blum, B. Gundlach, S. Mühle, J. M. Trigo-Rodriguez, Comets formed in solar-nebula instabilities!-An experimental and modeling attempt to relate the activity of comets to their formation process. Icarus 235, 156-169 (2014). doi: 10.1016/ j.icarus.2014.03.016

\section{ACKNOWLEDGMENTS}

The authors are indebted to DLR, the Helmholtz Association (grant PD-106 to I.P.), the Polish Academy of Sciences, the Austrian Academy of Sciences, and the UK Science and Technology Facilities Council (grant ST/L000776-1 to A.H.) for funding and to ESA for providing the opportunity to be on this exciting mission. The data used in this letter are available through the ESA Planetary Science Archive www.rssd.esa.int/psa.

\section{SUPPLEMENTARY MATERIALS}

www.sciencemag.org/content/349/6247/aab0464/suppl/DC1 Materials and Methods

Figs. S1 to S6

Table S1

References (30-32)

3 March 2015; accepted 23 June 2015

10.1126/science.aab0464 